\title{
e-Migrinter
}

$5 \mid 2010$

Migrations dans les pays nordiques

\section{Habiter une ville lointaine : le cas des migrants} japonais à Paris

Thèse soutenue le 30 novembre 2009 à l'Université de Poitiers

\section{Hadrien Dubucs}

\section{OpenEdition}

Journals

\section{Édition électronique}

URL : https://journals.openedition.org/e-migrinter/1937

DOI : 10.4000/e-migrinter. 1937

ISSN : 1961-9685

\section{Éditeur}

UMR 7301 - Migrinter

\section{Édition imprimée}

Date de publication : 28 avril 2010

Pagination : 73-75

ISSN : 1961-9685

\section{Référence électronique}

Hadrien Dubucs, « Habiter une ville lointaine : le cas des migrants japonais à Paris », e-Migrinter [En ligne], 5 | 2010, mis en ligne le 20 avril 2020, consulté le 20 mai 2021. URL : http://

journals.openedition.org/e-migrinter/1937; DOI : https://doi.org/10.4000/e-migrinter.1937 


\section{Habiter une ville lointaine : le cas des migrants japonais à Paris}

\section{Thèse soutenue par Hadrien Dubucs le 30 novembre 2009 à l'Université de Poitiers}

C ette thèse a pour un objet un exemple de migration entre métropoles de pays du Nord (la mégalopole japonaise et la métropole parisienne), et qui est le fait d'individus qui, pour leur grande majorité, ont un haut niveau de qualification et disposent de revenus économiques élevés. Les 10 à $20000^{1}$ Japonais résidant à Paris se répartissent en trois groupes: les actifs employés par des sociétés et administrations japonaises et qui séjournent deux à cinq ans à Paris, accompagnés de leur famille, dans le cadre d'une expatriation; les étudiants (environ 2500 entrées par an depuis les années 1990), pour qui l'attractivité de Paris relève de certains domaines précis: le français bien sûr, l'artisanat d'art, la cuisine et la pâtisserie, la musique; enfin, un ensemble hétérogène de migrants d'âge actif, qui dans certains cas arrivent à Paris en tant qu'étudiants, dont la migration se fonde sur des motifs existentiels: changer de vie, expérimenter un environnement urbain, social ou professionnel inédit, prendre ses distances avec un entourage jugé étouffant, etc. Ce type d'aspirations peut se concrétiser, pendant le temps du séjour parisien, par l'exercice d'activités professionnelles précaires ou relativement peu qualifiées (serveurs, employés de bureau dans les filiales parisiennes de sociétés japonaises). C'est également à cette sous-population qu'appartiennent les professions indépendantes, artistiques notamment.

\footnotetext{
1 Le Recensement Général de la Population de l'INSEE donne pour 1999 le chiffre de 10000 immigrés japonais résidant en Ile-de-France, dont 6 000 à Paris intra muros. L'ambassade du Japon produit ses propres statistiques et estime pour octobre 2003 à 22800 le nombre de Japonais résidant en Ile-deFrance, dont près de 15000 à Paris intra muros.
}

Par leur niveau de qualification, et étant originaires de l'un des pays les plus riches du monde, les migrants japonais séjournant à Paris relèvent de ce que l'on peut qualifier une "élite migrante", à condition de donner à ce terme une extension relativement large, qui dépasse les seules transnational elite (cadres de la finance notamment) qui dans les années 1990 ont focalisé l'attention des chercheurs s'intéressant aux mobilités induites par la mondialisation économique et la métropolisation (Sassen, 1991 : Beaverstock, 2002). Alors que ces travaux ont surtout appréhendé les élites migrantes de manière agrégée, en mettant en relation leurs flux migratoires avec les flux d'investissements à l'étranger, ou en décrivant la géographie résidentielle originale des migrants qualifiés dans les villes de séjour (White, 1998), je me suis attaché à analyser les manières individuelles d'habiter la métropole parisienne. Par manières d'habiter, j'entends l'ensemble des rapports pratiques (agencement spatial des activités banales ou plus rares) et idéels (représentations et discours relatifs à leur environnement urbain) que les individus entretiennent avec leur espace de séjour. Abordée à l'échelon des individus, la migration du Japon à Paris constitue une expérience de confrontation à de multiples déclinaisons de la grande distance spatiale: distance kilométrique entre la France et le Japon, qui se traduit par de fortes distance-temps et distance-coût; disparités entre contextes urbains (logement, distances des déplacements quotidiens, services urbains); contraintes pratiques liées à une faible compétence en français ou à la méconnaissance de l'espace de résidence.

L'hypothèse abordée dans cette recherche est donc que les manières individuelles d'habiter en situation de 
migration relèvent de stratégies pour «faire avec» la distance inhérente à cette expérience de mobilité. Cette réflexion s'inscrit dans deux questionnements plus larges sur les migrations internationales. Le premier porte sur les articulations entre pratiques citadines et expérience migratoire: quelles sont les interrelations entre les manières d'habiter l'espace de séjour, les rapports (physiques, communicationnels ou affectifs) avec les autres lieux de l'espace de vie, les projets personnels associés au séjour, ou encore la trajectoire migratoire antérieure? Le second porte sur le cas plus particulier des élites migrantes entre métropoles internationales: quelles formes prend la confrontation à la distance, et quelles sont ses traductions dans les manières d'habiter, dans le cas d'une population à propos de laquelle a pu être développée l'idée d'un mode de vie «anational» (en termes de système de valeurs, de composition des réseaux sociaux) et d'une mobilité fluide entre des contextes résidentiels relativement similaire d'une métropole à l'autre?

D'un point de vue méthodologique, cette recherche met en œuvre une approche compréhensive, dynamique et multi-scalaire des manières d'habiter. Le principal matériau empirique est issu d'une série d'entretiens approfondis avec trente-sept migrants japonais de profils variés, que j'ai rencontrés pour la plupart à plusieurs moments de leur séjour à Paris. Cette approche qualitative est complétée par le traitement statistique et cartographique de données détaillées de recensement (INSEE), ainsi que par une série d'enquêtes ciblées : entretiens avec des professionnels de l'immobilier à Paris et avec des acteurs politiques parisiens ; observation dans des secteurs de concentration japonaise ; observation à Tokyo et à Osaka des lieux habités par les enquêtés avant leur séjour parisien. Le croisement de ces différentes sources d'information vise à décrire en détail les manières individuelles d'habiter Paris, à les situer au sein d'un système de contraintes et de ressources plus structurelles, et de les inscrire dans une perspective plus large, temporelle (la trajectoire biographique de l'individu, dont la «séquence» parisienne n'est que l'une des composantes) et spatiale (l'espace de vie).

L'analyse de ce matériau montre d'abord que, par leurs manières d'habiter Paris, les migrants japonais étudiés prennent en charge cette confrontation à la distance de façon dynamique, complexe et différenciée. Les temporalités du séjour et les significations attribuées à la séquence parisienne des trajectoires biographiques apparaissent comme des facteurs-clés de différenciation, et se traduisent dans les choix résidentiels à Paris comme dans les échanges avec les lieux japonais de l'espace de vie. Ainsi, les catégories socioprofessionnelles usuellement mobilisées n'éclairent que très imparfaitement les manières d'habiter, et doivent être complétées par des catégories plus compréhensives. En reprenant des travaux d'anthropologues sur la multi-résidence (Rémy, 1999), j'ai ainsi proposé une typologie des individus selon que Paris apparait comme une "étape», un "espace de primarité », un «espace d'expérimentation", ou un "espace de vie par alliance ». Ces significations éclairent aussi le rythme et l'ampleur de l'autonomisation des migrants japonais vis-àvis des ressources commerciales, sociales ou informationnelles qui leur sont dédiées. A cet égard, le cas des migrants japonais illustre clairement les liens entre l' «autonomie» comme capacité d'initiative individuelle (fondée sur les capitaux sociaux et économiques mobilisables par les individus) et l' "autonomie» comme stratégie d'identification de soi à un groupe d'appartenance (Ma Mung, 2009). Enfin, par leurs manières d'habiter, ces migrants contribuent à certaines dynamiques urbaines : renforcement de l'image internationale de quartiers valorisés du centre-ouest parisien; spécialisation d'un segment du parc locatif dans l'accueil d'étrangers qualifiés; complexification des 
marquages sociaux dans des quartiers en cours de gentrification; création d'une offre commerciale et culturelle spécifique qui, dans le quartier de l'Opéra, enrichit l'attractivité d'une centralité économique et historique majeure de la métropole. Ces contributions reposent sur la position ambivalente des migrants japonais à Paris, entre proximité liée à leur statut d'élites migrantes originaires d'un pays du Nord, et distance inhérente à une migration lointaine.

Hadrien Dubucs Docteur en géographie MIGRINTER - UMR 6588 CNRS / Université de Poitiers hdubucs@gmail.com
Bibliographie

Beaverstock, Jonathan V. (2002) Transnational elites in global cities: British expatriates in Singapore's financial district, Geoforum, vol. 33, n4 pp. 525-538.

Ma Mung, Emanuel (2009) Le point de vue de l'autonomie dans l'étude des migrations internationales : «penser de l'intérieur» les phénomènes de mobilité, in Dureau, F.; Hilly, M.-A. (Dir.), Les mondes de la mobilité. Exploration d'un paradigme, Presses Universitaires de Rennes, pp. 13-28.

Remy, Jean (1999) Dédoublement des espaces sociaux et problématiques de l'habitat, in Bonnin, P.; Villanova de, R., D'une maison l'autre, parcours et mobilités résidentielles, Grane, éditions Créaphis, pp. 315-345.

Sassen, Saskia (1991) The Global City. New York, London, Tokyo, Princeton, Princeton University Press, 398 p.

White, Paul E. (1998) The settlement patterns of developed world migrants in London, Urban Studies, 35, pp. 1725-1744. 